\title{
Field Trial of Networked Social Robots in a Shopping Mall
}

\author{
Masahiro Shiomi, Takayuki Kanda, Dylan F. Glas, Satoru Satake, Hiroshi Ishiguro, Norihiro Hagita
}

\begin{abstract}
This paper reports the challenges of developing multiple social robots that operate in a shopping mall. We developed a networked robot system that coordinates multiple social robots and sensors to provide efficient service to customers. It directs the tasks of robots based on their positions and people's walking behavior, manages the paths of robots, and coordinates the conversation-performance between two robots. Laser range finders were distributed in the environment to estimate people's positions. The system estimates such human walking behaviors as "stopping" or "idle walking" to direct robots to provide appropriate tasks to appropriate people. Each robot interacts with people to provide recommendation information and route information about shops. The system sometimes simultaneously uses two robots to lead people from one place to another. The field trial, which was conducted in a shopping mall where four robots interacted with 414 people, revealed the effectiveness of the network robot system for guiding people around a shopping mall as well as increasing their interest.
\end{abstract}

\section{INTRODUCTION}

$\mathrm{W}$ ITH the advance of robotics technologies, researchers have started to explore the application of social robots to our daily life. Previous studies have revealed that social robots can be used as museum guides [1,2], as receptionists for assisting visitors [3], as peer-tutors in schools [4], in the context of mental-care for elderly people [5], in autism therapy $[6,7]$, and in child-care [8].

One crucial challenge is to operate such robots in a public place, e.g., shopping malls, where people do not necessarily approach the robot, but instead the robot proactively approaches people to offer an information providing service. In our previous study, we revealed how such a robot could be used in a shopping mall [9]; however, in that situation, a robot was placed in a mall, and interested people had the choice to approach the robot.

Moreover, we wonder about future cities with such social robots. Probably more than one robot will operate in such environments. Will they cooperate together? How can that be accomplished? How will people interact with such robots? To answer these questions, we developed a prototype system that consists of multiple social robots and conducted a field trial in a shopping mall.

In this paper, we report a number of technical challenges

Manuscript received March 1, 2009. This research was supported by the Ministry of Internal Affairs and Communications of Japan

M. Shiomi, T. Kanda, D. F. Glas, S. Satake, H. Ishiguro, and N. Hagita are with the Advanced Telecommunications Research Institute International, Kyoto, JAPAN (corresponding author to provide phone: +81-774-95-1432; fax: +81-774-95-1401; e-mail: m-shiomi@atr.jp).

H. Ishiguro is also with Osaka University, Osaka, Japan. for multiple networked social robots in a shopping mall (Fig. 1). The laser range finders placed in the environment track visitor positions, and a network robot platform directs the four robots' tasks and coordinates locomotion paths as well as conversation scenarios between robots. The robots provide recommendation information about shopping, route guidance, and invite/lead visitors to specific shops. The robots are partially semi-autonomous due to the difficulty of speech recognition; a technique for teleoperation is being studied [10].

\section{RoBOT's TASK IN A SHOPPING MALL}

What kind of robots do people want in their daily lives? According to a Japanese government report [11], a majority believe that providing information at such public spaces as stations and shopping malls is one desired task of robots. People also want robots to perform physical tasks, such as toting luggage.

Therefore, we decided to explore an information-providing task in a public space for a guide robot at a mall with many shops nearby. Guiding and leading are also promising tasks in a mall. In navigating tasks, our designed system sometimes simultaneously uses two robots to lead people from one place to another place to execute the tasks more efficient by handing over the tasks to the robots. Our design also allows robots to proactively approach people to more efficiently provide services.

\section{A. Proactive Approach}

Approaching is a basic function for mobile robots. In such public spaces as a mall, a robot's existence is still novel for most people. Therefore, robots can easily find people with whom to inter and for whom to provide services by approaching them. Particularly, window shoppers might welcome such additional information provided by a robot.

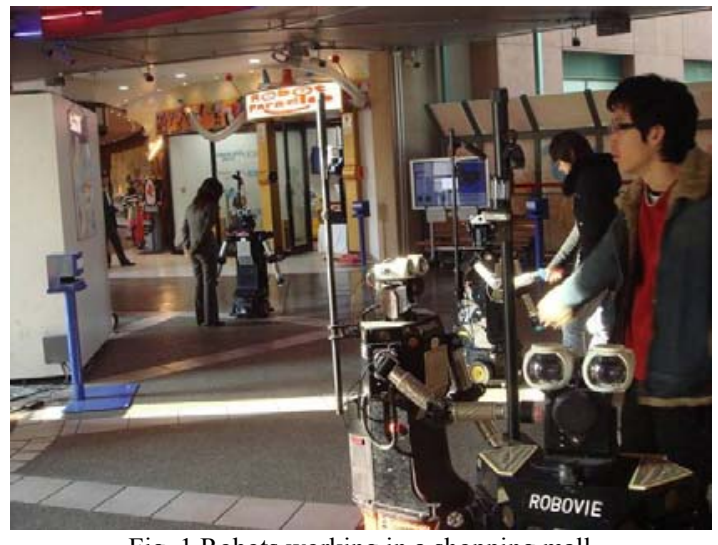

Fig. 1 Robots working in a shopping mall 
Moreover, proactive approaching is one important function for a robot in a shopping mall because it enables robots to increase interaction opportunities with people, who are interested in robots, have some free time, and/or need such help as route guidance.

\section{B. Recommendations}

From the mall's view, recommendations are crucial to attract visitor interests and to sell products to them. We believe that a robot can powerfully serve this purpose. Since a robot's presence is still novel for most people, it can attract people's attention and redirect their interest to the information it provides [12]. Other work has indicated the effectiveness of recommendations by a robot in malls [9].

\section{Route guidance}

From visitor views, route guidance is helpful in a large scale environment. Sometime people get lost in a big mall; they might ask for directions in such situations. People still prefer to ask for help even though a mall has maps because some information is not shown [9].

The different points between a robot and a map or other facilities are physical existence, co-located with people, and equipped with human-like body properties. Thus, (Fig. 2) a robot can naturally explain a route like humans by pointing and using such reference terms as "this way." Such tasks quite effectively help people who are lost or looking at maps in a mall.

\section{Navigation}

In a shopping mall, robots need not only perform route guidance but also navigation for which mobile robots have an obvious advantage; they can lead a person to a specific shop by walking together and describing the shop if the person wants to visit it. Moreover, by lingering around the shop, the robot easily finds people interested in it. Therefore, two ways exist for navigation tasks: walking together and waiting.

In this study, we designed our system to recommend a specific shop and lead people to it if they want to visit. We also designed our system so that it simultaneously uses two robots to lead people from one place to the specific shop because, in navigation tasks, using two robots is more efficient than just one. For example, when a robot is waiting around the shop, the other robot can deliver the people being led to the waiting robot after navigation. The former robot can reduce the wasted time by assuming the task. The latter robot can start its next task without explaining the shop. Such collaboration is more novel than being led by one robot.

\section{Sensing People’s Position And BeHAVIOR}

We decided to use multiple laser range finders to robustly support robot sensing because they accurately and simultaneously identify the positions of multiple robots and people. The robot's position information is used to localize each robot and ensure safety. The people's position information is used to estimate such behaviors as stopping, walking, and running; such trajectory-related information of people is one appropriate criterion to decide robot tasks. Moreover, we often observed that a person "walking slowly," "stopping around the map," or "approaching the robot" is an appropriate target for whom the robot can furnish an information-providing service. We considered three essential types of trajectory-related information: local behavior, spatial behavior, and global behavior.

\section{A. Position}

We installed multiple SICK LMS-200 LRFs around the environment's perimeter at a height of $85 \mathrm{~cm}$ (Fig. 3) to track people's positions. We used a technique derived from the algorithm presented in a previous work [13], in which individual particle filters were used to track the location of each person in the scanning area based on the combined torso-level scan data from all LRFs. This tracking technique provides highly stable and reliable trajectory data (Fig. 4) For natural walking speeds, the tracking accuracy for our sensor configuration was measured to be $+/-6 \mathrm{~cm}$.

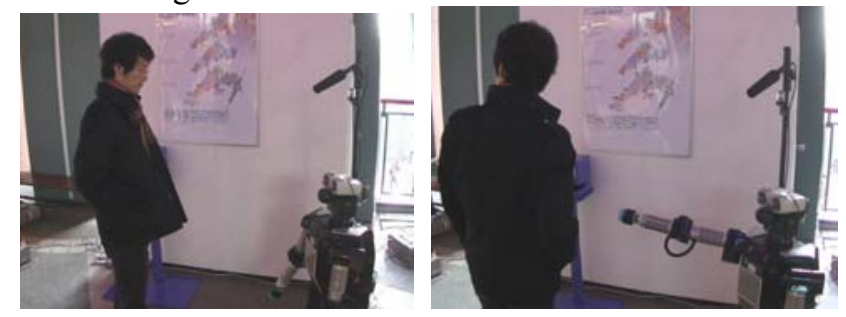

Fig. 2 Route guidance around the map

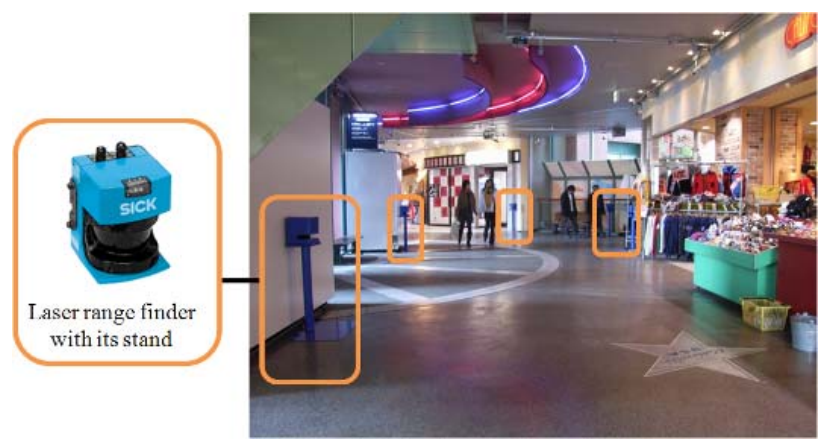

Fig. 3 Shopping arcade and laser range finders

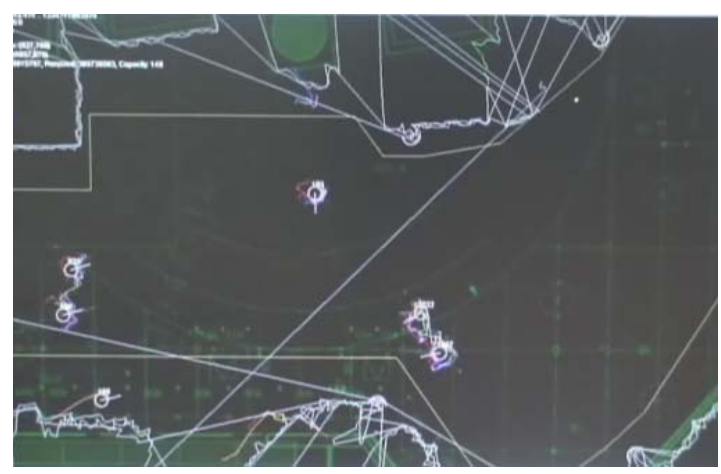

Fig. 4 Position estimation system
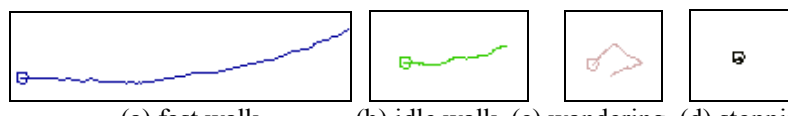

(a) fast walk

(b) idle walk (c) wandering (d) stopping

Fig. 5 Example of trajectories for local behaviors 


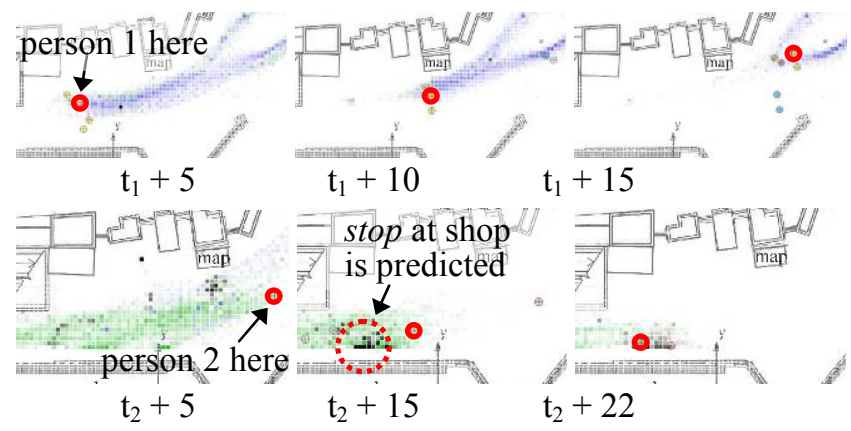

Fig. 6 Example of prediction of future local behavior

To track the robot positions in the environment, we used the system presented in [14], which associates all the robot odometry data with the observed trajectory of an entity detected by the laser tracking system. Kalman filters are used to correct the rotational offsets between the robots' individual coordinate systems and the global reference frame, enabling the robots to benefit from the shared information about the locations and the interaction histories of people in the environment.

\section{B. Local Primitive}

We defined the term local behavior to refer to basic human motion primitives, such as walking, running, going straight, and so on. We began our analysis with a classification system [15] that uses support vector machine (SVM) to categorize trajectories based on their velocity, direction, and shape features. Such features estimated by the position information from LRFs in the environment are used for learning. Currently, the classification system computes local primitives with their own SVM classifier. In this study, we used four types of local behaviors: fast walking, idle walking, wondering, and stopping (Fig. 5). We labeled 701 pieces of data and tested the system with the leaving-one-out method; classification accuracy averaged over $90 \%$.

\section{Spatial primitive}

Spatial primitives are used for defining the area's state that depends on such environment elements as the front of a map on a wall or a shop, a bench, and so on. The system classifies people's spatial primitives using $x-y$ coordinate information, which enables it to simplify the directing services of robots based on location information.

\section{Global Primitive}

Based on the accumulated trajectories, we analyzed how people visited the mall. We defined the term global behavior to refer to the overall trajectory patterns comprised of several local behaviors in sequence, such as "entering through the west entrance, walking across a street, and stopping at a shop." Global behaviors are highly dependent on environments.

Estimating people's global behavior enables us to easily make rules for task selection because global behavior can be used to predict their local behaviors a few seconds in the future. In particular, this information is useful for directing approaching tasks to the robot and setting such approach target local behavior as "stopping" or "idle walking."

To estimate global behaviors in the mall, we used a technique derived from the algorithm presented in a previous work [16], in which a clustering technique was applied to the accumulated trajectories to extract information. This technique enables the system to estimate the future local behavior of people as "stopping" and "idle walking" in the environment (Fig. 6).

\section{Network Robot System}

For robots working in our daily lives, we need to explore the promising combination of hardware and infrastructure. Some researchers are studying stand-alone robots that have complete sensing, decision making, and acting capabilities. In contrast, some are focusing on a combination of robots, ubiquitous sensors, and humans. We have chosen the latter strategy, known as a "network robot system" [17], in which a central system directs the tasks of multiple robots, ubiquitous sensors support robot sensing, and a human operator processes each robot's decisions during interaction.

Figure 7 shows an overview of a networked robot system that consists of three components: a network robot platform, a sensor infrastructure, and robots. Here we describe the details of the network robot platform. This platform manages multiple robots to provide services effectively to people in real environments; we believe it is the one of unique points as compared with previous works. The sensor infrastructure details were already described above, and the robot details are described in the next section.

\section{A. Directing Tasks}

The platform directs each task of each robot using pre-implemented rules that consist of the position relationships between robots and people, predictions of their local behaviors, and the abilities of the robots.

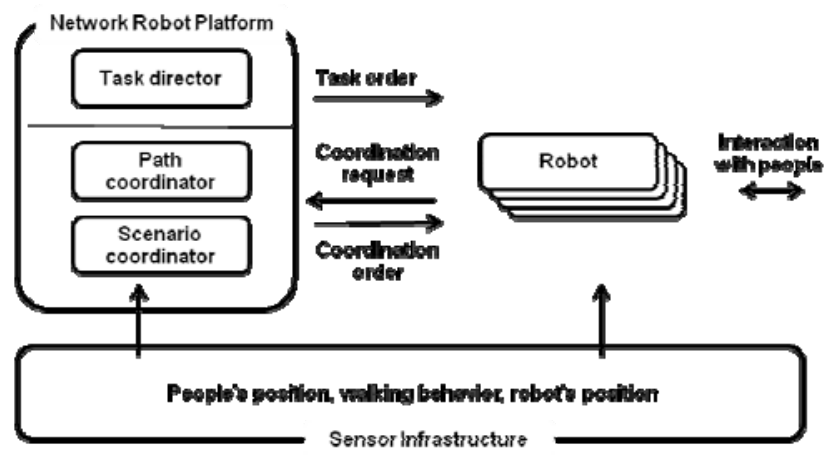

Fig. 7 Overview of networked robot system

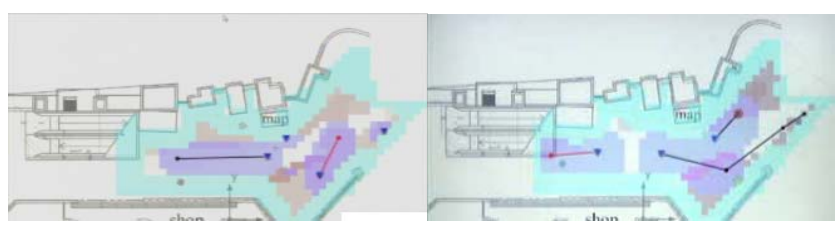

Fig. 8 Examples of path information 
The position relationships between robots and people are computed using position information from the sensor infrastructure. This criterion decides which robot should approach the people and wait around the map and the shop. For example, the waiting task around the map is directed to the robot nearest the map. Similarly, the waiting around the shop task is directed to the robot nearest the shop.

The predicted local behaviors are computed by estimated global behaviors, as described above. This criterion decides a target to approach. For example, people in "stopping" or "idle walking" local behaviors are appropriate targets to approach, but not "fast walking" local behavior because the robots should not interfere with busy people.

Such abilities of each robot as executable tasks are sent to the network robot platform when the system and the robot are connected. This criterion selects a robot with the ability to achieve the task. In this study, since we used the same robots, their properties are not different; however, such information must be collaborated with different kinds of robots in the future.

The tasks of waiting around the map and in the front of the shop are divided between the robots. The approaching task is directed to the two robots. The details of the pre-implemented rules are listed below:

- If a visitor's global primitive is estimated as "idle walking" or "stopping" and a robot's task is not assigned, the task director assigns the approaching task to the robot and sets the visitor as the target.

- If the waiting around the map task is not assigned, the task director assigns that task to the robot nearest the map.

- If the waiting in front of the shop task is not assigned, the task director assigns it to the robot nearest the shop.

- If a visitor wants to visit the shop when a robot is idle that has the task of waiting in front of the shop, the task director assigns the task of explaining the shop to the robot. In such situations, each robot starts to communicate by implemented language.

\section{B. Coordinating Paths}

The platform coordinates locomotion paths based on the coordinate requests from the robots. These requests are executed based on directed tasks. Each path is adjusted to avoid conflict between robots that have sent requests to the platform. Moreover, each path has a priority based on tasks; for example, an approaching path is given higher priority than a roaming path. In other words, the platform coordinates a path so that the roaming robot avoids an approaching robot.

After receiving paths, each robot autonomously starts to move based on the paths. Moving paths are computed beforehand based on measured location information and position information from the environmental sensors. We used the algorithm presented in [16] to adjust the moving paths. All paths are re-calculated when the positions of the robots or the people change (Fig. 8).
The destination of each path depends on the task. In the approaching task, the destination is set to the target location. In the waiting around the map task, the destination is set to the front of the map. In the waiting in the front of the shop task and the guiding to a shop task, the destination is set to the shop's front. If a robot has no task, a roaming path is set so that the robot moves around.

\section{Showing Conversation of Robots}

In this study, the system simultaneously uses two robots to lead people from one place to another; the robots talk with each other when one robot hands its task to another robot. To realize such autonomous conversation, we use our scripting language for multi robots [12] because it has adequate capabilities for describing multi-robot communication and is simple enough for developer to easily use it to control the robot behaviors. In this system, a set of robots interpret script files and execute scripts written in this language. One robot becomes the master; the platform set the navigating robot as a master in this study. The master robot sends a signal to its partner robot to control the conversation timing.

Figure 9 shows an example of collaboration with two robots in a navigation task. If a visitor wants to visit the shop recommended by robot-A, robot-A waves robot-B who is waiting around the shop (Fig. 9-a). Then robot-A leads the visitor to the front of the shop where robot-B waits for them (Fig. 9-b). Robot-A says, "I'll leave it up to you," and then robot-B says "Thank you. Hi, I'd like to tell you about this shop, please stand in front of me!" Then robot-A leaves the visitor with robot-B and continues to move around to provide services to other visitors (Fig. 9-c). Robot-B explains the shop to the visitor and recommends entering it (Fig. 9-d). If robot-B has already started its explanation or a conversation with someone, robot-A leads the visitor to the front of the shop and starts its explanation.

\section{IMPLEMENTATION OF ROBOTS}

For this study, we prepared four robots to provide services to visitors in the mall. We believe that installing four robots in the environment is sufficient because the environment has two suitable locations for waiting behaviors, a map and in the front of the shop, and the main corridor has enough space for the two robots to move around. Further, we used robots with the same appearance to avoid effects from different appearances.

\section{A. Hardware of the Robot: Robovie}

"Robovie" is an interactive humanoid robot characterized by its human-like physical expressions and its various sensors [18] (Fig. 10). It has a head, two arms, a body, and a wheeled-type mobile base. Its height and weight are $120 \mathrm{~cm}$ and $40 \mathrm{~kg}$, respectively. The robot has the following degrees of freedom (DOFs): two for the wheels, three for its neck, and four for each arm. We used a corpus-based speech synthesis [19] for generating speech. Robovie can work one hour without being recharged. Since it uses hot swap batteries, its 
batteries can be changed without shutdown in five to ten minutes.

Its lower mobile base is a Pioneer 3-DX (ActiveMedia). The maximum moving speed was set to $2.5 \mathrm{~km} / \mathrm{h} \quad(700$ $\mathrm{mm} /$ second), based on the average walking speed of people in a mall, the mobile base's capability, and safety concerns. Robovie has a Hokuyo URG-04LX laser range finder (LRF) to detect obstacles. The attached LRF enables the robot to detect low obstacles that the environmental sensors cannot. For example, if a child runs too close to the front of the robot, the robot can stop immediately using the attached LRF.

\section{B. Robovie's Software}

Basically, Robovie autonomously interacts with people based on position information obtained from environmental sensors and its own sensors. For example, the robot autonomously moves toward the target person and starts a conversation when an approaching task is directed. As in a WOZ method, speech recognition and correcting errors in interaction are conducted by a human operator. This information is sent to a behavior selector of Robovie, which chooses an interactive behavior based on pre-implemented guidelines called Episode Rules.

Interactive behaviors are implemented with situated modules ("behavior" in this paper) and episode rules [18]. The behavior transition is handled by a "behavior selector." Only one behavior is executable at each moment. After each execution of the behavior, the behavior selector chooses one of the behaviors based on the pre-implemented episode rule.

We set two basic policies for designing the robot's interaction behavior. First, it takes the communication initiative and introduces itself as a guide robot. It asks about places and then provides information in response to user requests. Thus, customers clearly understand that the robot is engaged in route guidance. Second, its way of utterance and other behaviors are prepared in an affective manner [20], not in a reactive manner. This is very different from master-slave type communication where a robot prompts a user to provide a command.

In route guidance behaviors, the robot explains a route to a destination with utterances and gestures (Fig. 2). The robot points in the first direction and says, "Please go that way," with an appropriate reference term chosen by an attention-drawing model [21]. It continues the explanation: "After that, you will see the shop on your right." Since the robots know all of the mall's shops and facilities (toilets, exits, nearest train station, etc.), they can explain 70 destinations.

We used a human operator for speech recognition and decision making during interaction. For this way of providing information, instability and awkwardness cause critical disappointment, and the quality of current speech recognition technology remains far from useful. For instance, a speech recognition system prepared for noisy environments, which provided $92.5 \%$ word accuracy in 75 dBA noise [22], resulted in only $21.3 \%$ accuracy in a real environment [23]. This reflects the natural way of daily utterances, the changes of voice volume among people and/or within the same person,

and the unpredictability of noise in real environments. Thus, since the speech recognition program causes too many recognition errors, the robots have to ask for elucidation too often.

\section{Teleoperation System for Multi Robots}

In this study, one operator supports the speech recognition of four robots, corrects navigation errors, and supervises the monitoring of the robots to detect other kinds of errors. In particular, speech recognition is important to realize smooth interaction between robots and people. Correcting navigation errors is dealt with when the tracking robot function failed due to occlusions caused by too many people.

For the teleoperation of the four robots, we used an interface that displays sensor information from each robot and the environment [13] (Fig. 11). The operator can listen to visitor responses to the robot questions and choose the appropriate button as well as the speech recognition function of the robots. The operator can also correct navigation errors through the interface.

One problem in teleoperation with multiple conversational robots is the conflict of using the operator's resources. The operator can only deal with speech recognition for one robot at the same time, even though multiple robots simultaneously need the resource. Conversational interactions tend to follow patterns that sometimes make it possible to anticipate the need for the operator. We used a technique to schedule behaviors to avoid conflicts over the operator resources [13].

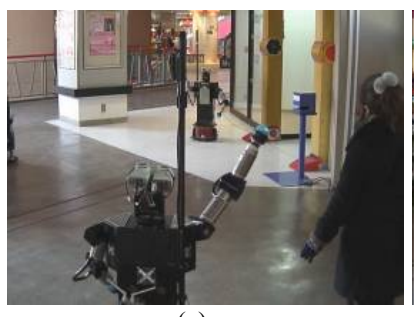

(a)

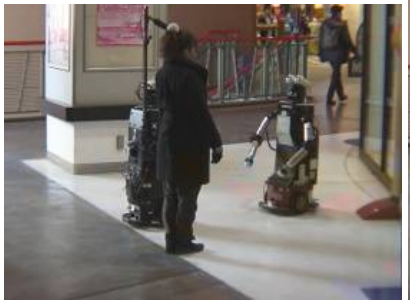

(c)

Fig. 9 Scenes of collaboration with two robots

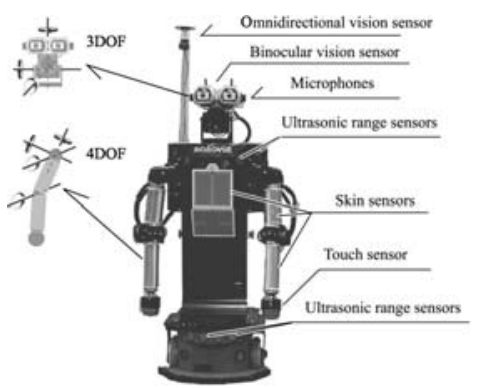

Fig. 10 Robovie

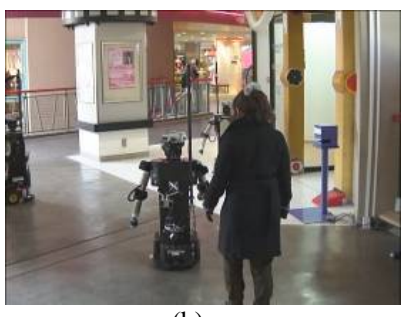

(b)

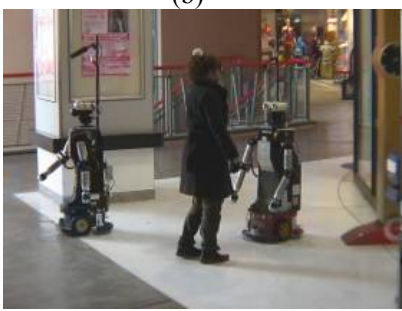

(d) 


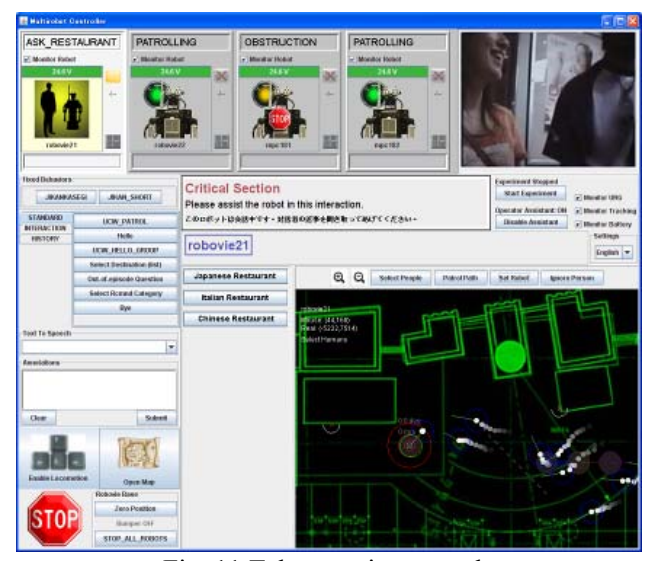

Fig. 11 Teleoperation console

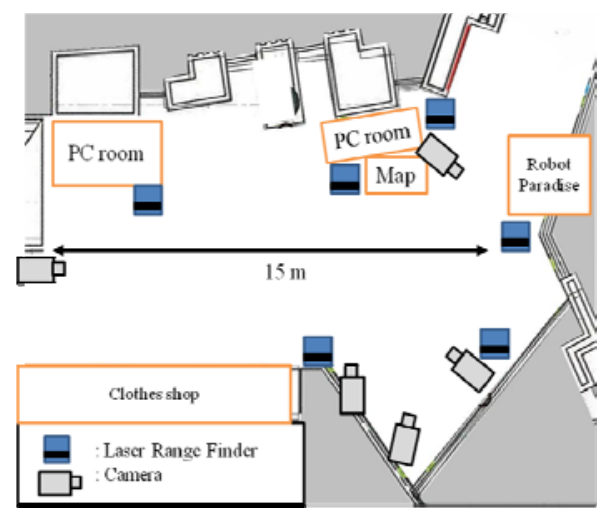

Fig. 12 Map of mall

\section{FIELD TRIAL}

\section{A. Environments and Settings}

The field trial was conducted at a mall located between a train station and an amusement park. The robots were placed in a major corridor of the mall. Fig. 12 shows the environment, where we installed six LRFs and five cameras.

Visitors to the mall were mainly families, couples, and sightseers who could freely interact with the robot. The number of visitors was different between day and night, so we divided the experiment time into time slots that each covered both daytime (when people mainly go to lunch, shopping, or the amusement park) and night (when people mainly go to dinner, shopping, or the station) to avoid skewed results due to the difference in the number of participants.

In the trials, we used one operator for the teleoperation of the speech recognition of the four robots. In addition, one staff member stayed around the robots for safety. ${ }^{1}$

\section{B. Results}

Since our purpose sought to verify the system's basic performance, we conducted a field trial for six hours on a weekend. This section reports the results from four viewpoints: how the system worked, how visitors interacted

\footnotetext{
${ }^{1}$ We obtained permission to record video and sensor data from the responsible authorities of the mall. The experimental protocol was reviewed and approved by our institutional review board.
}

with the robots, how they perceived the robots, and how the robots affected visitor behaviors.

\section{1) System Performance}

The network robot platform worked quite well. It correctly directed four robot tasks and coordinated locomotion paths as well as a conversation scenario between robots. Figure 13 shows an example scene where the task director and the path coordinator worked well. The system directed approaching tasks to the robot (second from right) when the system detected an "idle walking" person at the front of the shop. At the same time, the path coordinator organized the robot's locomotion path (extreme right) to avoid a conflict. Then the robot (second from right) started to interact with the person.

The scenario coordinator also worked well in the trials. As shown in Fig. 9, navigation services (inviting/taking a person to the shop) are usually conducted with two robots: one leads the person while the other waits in front of the shop. In this situation, the scenario coordinator correctly adjusted the timing of the conversation and the gestures of the robots; therefore, two robots smoothly talked with each other.

Figure 14 shows other example scene where the task director assigned navigation tasks to a robot by considering the states of other robots. In this scene, a robot waiting in front of the shop is interacting with a family. Other robots are also interacting with visitors. Therefore, the task director assigned navigation service to one robot. The path coordinator orchestrated the robot's locomotion path to approach the shop by avoiding the family, so that the robot stopped at a place slightly far from the shop. After stopping, the robot started to explain the shop to the visitor.

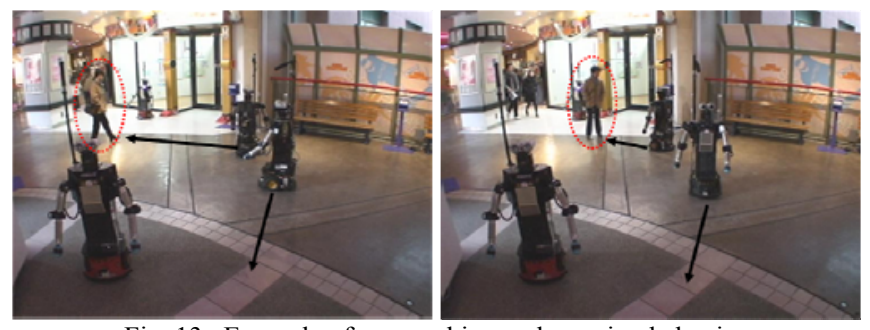

Fig. 13 Example of approaching and roaming behavior

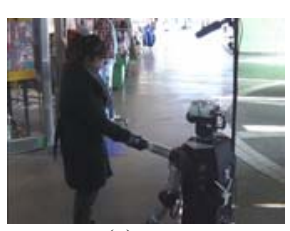

(a)

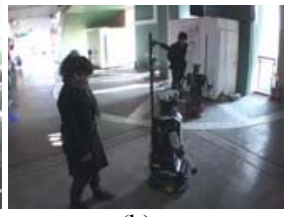

(b)

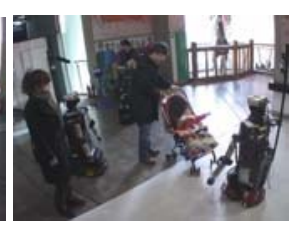

(c)
Fig. 14 Example of navigating behavior by one robot

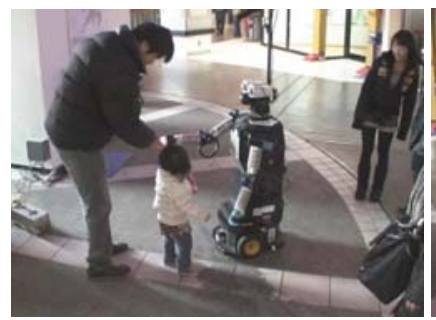

(a)

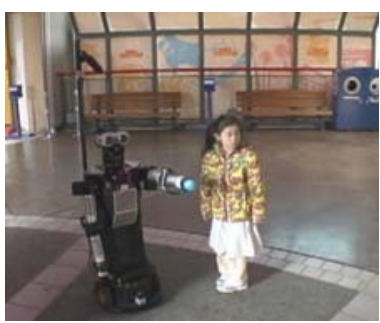

(b)
Fig. 15 Observed interaction scenes in trials 


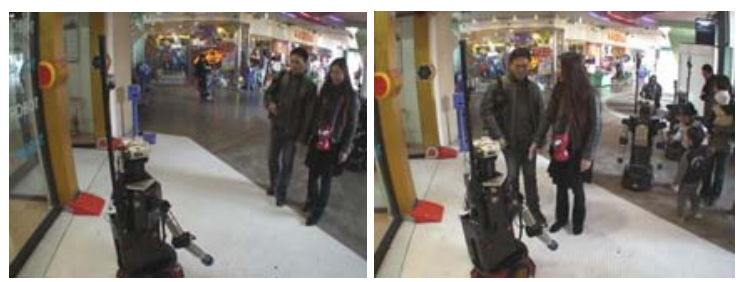

Fig. 16 Two persons interested in the robot and the shop

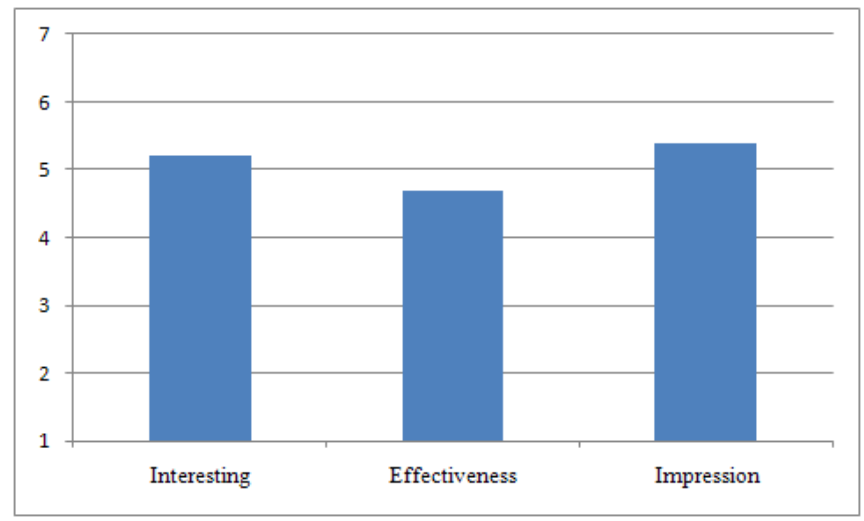

Fig. 17 Questionnaire results

Note that sensors robustly estimated the positions of people and robots in crowded situations; for example, in the trials at night, even though many occlusions were caused by the presence of over 20 people, none of the robots collided with anyone. The attached LRFs of the robots were also helpful to detect obstacles in such situations. Therefore, robots never caused a dangerous situation (e.g., with small children, the elderly, or the handicapped).

The operator also worked well as a speech recognition function of the four robots in the trials. Scheduled behaviors enabled the operator to efficiently support all robots. For example, during crowded situations, the four robots were sometimes simultaneously interacting with visitors. They smoothly talked with the visitors with the operator's support during such situations. Moreover, the operator sometimes corrected navigation errors in crowded situations.

\section{2) Visitor Interactions with the Robot}

Visitors freely interacted with the robot (Fig. 15-a). Many people were curious about it without interacting in any depth. They observed how the robot behaved while providing minimum response. For example, when the robot offered to shake hands, most visitors assented, and then some immediately said goodbye. When a robot offered route guidance, some visitors asked for a simple destination such as a station or the toilet but the visitors did not talk too much. They seem satisfied after a brief interaction with the robot. A certain amount of people only observed how the robots behaved and interacted with others, but they did not directly interact with the robots. Such bystanders have often been observed in past field trials $[12,23]$; they seemed satisfied by observation only.

Perhaps, more interesting interactions reflect the smaller numbers of people who actually used the robots. Some visitors asked for information about a place that they really seemed to want to find, such as a restaurant, a clothing shop, or a place to smoke. These people appeared satisfied with the information from the robots, said thank you, and left for the place after getting route guidance from the robot (Fig. 15-b). 3) Effect of Robot's Recommendation on Visitor Behaviors

In the trials, 2411 people visited the corridor, 414 of whom the robots interacted with. They successfully elicited interest from 46 of the 414 interacted visitors to look at the shop recommended by the robot (15 subsequently entered it). We defined a successful recommendation to be when the person looked at the shop for more than three seconds while stopping around $3 \mathrm{~m}$ from the shop's entrance.

Those who interacted with the robots received a recommendation to visit the "robot shop." Some visitors listened to the recommendation from the robots and went to the shop (Fig. 16). Unfortunately, the shop was not really a place to buy interesting goods, but merely an exhibition place with some posters on the wall (we failed to fully prepare for the field trial). Thus, naturally a limited number of people visited the shop. In fact, to investigate the effectiveness of robots' existence, we have also conducted a field trial for six hours on a weekend without the robots. In the trial, 2158 people visited the corridor. Only 19 of the visitors looked at the shop (anyone did not enter it).

4) Visitor Perceptions

We asked visitors to complete a questionnaire when they finished interacting with the robots. All items were on a 1-to-7 point scale where seven represents the most positive, four is neutral, and one represents the most negative. We received 103 questionnaires from visitors. The questionnaire included items about interest in the contents of the robot's conversation, the effectiveness of the robots, and impressions of the robots. Fig. 17 shows that all the questionnaire results were above average. In a free-description form, the following comments were made:

- These robots are cute and interesting.

- I'm interested in route-guidance by robots.

- The robot's recommendation got me interested in the shop, so I went in.

- I could easily understand the route guidance by the pointing gestures.

- The robots' conversation was interesting, but not the shop. I didn't see the shop before interacting with the robots

- It's interesting that the robot recognized my location and changed its direction to me.

- I found it interesting that a robot could wave its hand to signal another waiting robot, and then the waiting robot raised its hand in response.

\section{5) Summary}

We confirmed that our networked robot system correctly worked in a real environment through field trials. The network platform directed four robot's tasks based on pre-implemented rules and sensor information. Coordinated paths enabled the robots to smoothly move around in the environment. The platform sometimes used two robots for navigation tasks depending on the situations, which 
redirected visitors' interest to the shop. Questionnaire results indicated that almost all visitors had good impressions of the robots. Therefore, these results indicated the effectiveness of our system in a real environment. We believe that such a networked robot system is one appropriate approach to realize a future society that includes social robots.

\section{CONCLUSION}

We developed a networked robot system that coordinated four social robots and sensors to provide efficient service to visitors in a shopping mall. The networked robot system consists of three components: a task director, a path coordinator, and a scenario coordinator. The task director directed the robot tasks based on their positions and people's walking behaviors. The path coordinator managed the paths of robots based on position information. The scenario coordinator orchestrated the conversation-performance of two robots. Six laser range finders were installed in the environment to estimate the positions of people and robots. People positions were used to estimate such walking behaviors as "stopping" or "idle walking" to direct robots to provide appropriate tasks to appropriate people. Four social robots provided route guidance information and led people to a shop. Only one human operator supported these robots through an interface designed to simultaneously control multiple social robots.

A two-day field trial was conducted in a mall. We confirmed that our networked robot system correctly worked in a real environment. In the trials, 2411 people visited the corridor, and the robots interacted with 414 of them. Moreover, the robots attracted the attention of 46 visitors and redirected them to the shop through interaction; 15 subsequently entered the shop space. The questionnaire results show that visitors had positive impressions of the robots. We believe that our networked robot system indicated the effectiveness of using multiple social robots in real environments.

\section{ACKNOWLEDGMENTS}

We wish to thank the administrative staff at Sumisho Urban Kaihatsu Co., Ltd. for their helpful participation. We also wish to thank Dr. Akimoto, Dr. Miyashita, and Mr. Kurumizawa for their help.

\section{REFERENCES}

[1] W. Burgard, A. B. Cremers, D. Fox, D. Hänel, G. Lakemeyer, D. Schulz, W. Steiner, and S. Thrun, The interactive museum tour-guide robot, Proc. of National Conference on Artificial Intelligence, pp. 11-18, 1998.

[2] R. Siegwart et al., Robox at Expo.02: A Large Scale Installation of Personal Robots, Robotics and Autonomous Systems, 42(3), pp. 203-222, 2003.

[3] R. Gockley, J. Forlizzi, and R. Simmons, Interactions with a Moody Robot, ACM/IEEE International Conference on Human-Robot Interaction (HRI2006), pp. 186-193, 2006.

[4] T. Kanda, T. Hirano, D. Eaton, and H. Ishiguro, Interactive Robots as Social Partners and Peer Tutors for Children: A Field Trial, Human Computer Interaction, 19(1-2), pp. 61-84, 2004.
[5] K. Wada and T. Shibata, Living With Seal Robots - Its Sociopsychological and Physiological Influences on the Elderly at a Care House. IEEE Transactions on Robotics, 23(5), pp. 972-980, 2007.

[6] K. Dautenhahn and I. Werry, A quantitative technique for analyzing robot-human interactions, IEEE/RSJ International Conference on Intelligent Robots and Systems (IROS'02), pp. 1132-1138, 2002.

[7] H. Kozima, C. Nakagawa, and Y. Yasuda, Interactive robots for communication-care: A case-study in autism therapy, IEEE International Symposium on Robots and Human Interactive Communications (Ro-Man 2005), pp. 341-346, 2005.

[8] F. Tanaka, A. Cicourel, and J. R. Movellan, Socialization between toddlers and robots at an early childhood education center, Proc. of the National Academy of Sciences of the USA, 104(46), pp. 17954-17958, 2007.

[9] T. Kanda, M. Shiomi, Z. Miyashita, H. Ishiguro, and N. Hagita, An affective guide robot in a shopping mall, ACM/IEEE International Conference on Human-Robot Interaction (HRI2009), 2009. (to appear)

[10] D. F. Glas, T. Kanda, H. Ishiguro, and N. Hagita, Simultaneous Teleoperation of Multiple Social Robots, ACM/IEEE International Conference on Human-Robot Interaction (HRI2008), pp. 311-318, 2008.

[11] Research study for the scope of the strategy map of robotics technology, 2005. (Available at http://www.nedo.go.jp/ database/index.html, with index code 100007875) (in Japanese)

[12] K. Hayashi et al., Humanoid robots as a passive-social medium - a field experiment at a train station, HRI2007, pp. 137-144, 2007.

[13] D. F. Glas et al., Laser Tracking of Human Body Motion Using Adaptive Shape Modeling in Proc. Int. Conf. Intelligent Robots and Systems, pp. 602-608. 2007.

[14] D. F. Glas et al., "Simultaneous People Tracking and Localization for Social Robots Using External Laser Range Finders," IROS2009, Under review

[15] S. Nishio et al., Robotic Platforms Structuring Information on People and Environment, IEEE/RSJ International Conference on Intelligent Robots and Systems, 2008

[16] T. Kanda et al., Who will be the customer? A social robot that anticipates people's behavior from their trajectories, UbiComp2008, 2008

[17] A. Sanfeliu, N. Hagita, and A. Saffiotti, "Network Robot Systems," Special Issue: Network Robot Systems, Robotics and Autonomous Systems, 2008.

[18] T. Kanda, H. Ishiguro, M. Imai, and T. Ono, Development and Evaluation of Interactive Humanoid Robots, Proceedings of the IEEE, Vol. 92, No. 11, pp. 1839-1850, 2004.

[19] H. Kawai, T. Toda, J. Ni, M. Tsuzaki, and K. Tokuda, XIMERA: A New TTS from ATR Based on Corpus-Based Technologies, Proc. of Fifth ISCA Workshop on Speech Synthesis (SSW5), pp. 179-184, 2004

[20] R. W. Picard, Affective Computing, 1997

[21] O. Sugiyama et al. "Humanlike conversation with gestures and verbal cues based on a three-layer attention-drawing model," Connection science, 18(4), pp. 379-402, 2006.

[22] C. T. Ishi, S. Matsuda, T. Kanda, T. Jitsuhiro, H. Ishiguro, S. Nakamura, and N. Hagita, A Robust Speech Recognition System for Communication Robots in Noisy Environments, IEEE Transactions on Robotics, 24(3), pp. 759-763, 2008.

[23] M. Shiomi, D. Sakamoto, T. Kanda, C. T. Ishi, H. Ishiguro, and N. Hagita, A Semi-autonomous Communication Robot -A Field Trial at a Train Station -, ACM/IEEE International Conference on Human-Robot Interaction (HRI2008), pp. 303-310, 2008. 